\title{
TÉCNICA DE TUNELIZAÇÃO DO TESTÍCULO EXPOSTO E IMPORTÂNCIA DO CONHECIMENTO DA ANATOMIA NA GANGRENA DE FOURNIER
}

\author{
TECHNIQUE OF TUNNELING THE EXPOSED TESTICLE AND THE \\ IMPORTANCE OF KNOWLEDGE OF THE ANATOMY IN FOURNIER`S \\ GANGRENE
}

\author{
Rui Lopes Filho ${ }^{1}$ \\ Lucas Carvalho Lopes ${ }^{2}$ \\ Laura Janot Pacheco Muzzi Martins ${ }^{3}$ \\ Ana Beatriz Vianna Pedrosa ${ }^{4}$
}

RESUMO: Introdução: $O$ conhecimento da anatomia é importante no entendimento da progressão da gangrena de Fournier. A exposição dos testículos constitui um problema, após fasciite necrosante escrotal ou gangrena de Fournier, devido à possibilidade de redução da espermatogênese, sobretudo em jovens. Objetivo: Apresentar a técnica de tunelização do testículo exposto e a importância da anatomia na gangrena de Fournier. Método: Realizou-se a descrição da técnica de tunelização do testículo exposto e apresentou-se a importância da anatomia na gangrena de Fournier. Resultados: A técnica de tunelização do testículo proporcionou a cobertura com proteção do testículo exposto. As comunicações anatômicas entre as camadas de revestimento das regiões escrotal, perineal, peniana e abdominal, permitem a disseminação do processo infeccioso decorrente da gangrena de Fournier para as regiões adjacentes. Conclusão: A técnica de tunelização do testículo exposto na coxa possibilitou a proteção do testículo $e$ as comunicações entre as camadas de revestimento das regiões escrotal, perineal, peniana e abdominal contribuiu para a disseminação do processo infeccioso da gangrena de Fournier.

Descritores: Gangrena de Fournier. Fasciite Necrosante. Anatomia. Cirurgia Geral. Cirurgia Plástica.

\footnotetext{
${ }^{1}$ Doutor e Mestre em Cirurgia pela Faculdade de Medicina da Universidade Federal de Minas Gerais. ORCID: https://orcid.org/oooo-ooo2-1627-937I E-mail: ruilopesfilho@terra.com.br

2 Graduação em Medicina da Faculdade de Ciências Médicas de Minas Gerais ORCID: https://orcid.org/oooo-ooo2-0445-1330E-mail: carvalholopesl@gmail.com

3 Graduação em Medicina da Faculdade de Ciências Médicas de Minas Gerais. ORCID: https://orcid.org/oooo-ooo3-2348-2770 E-mail: laura.janotor@gmail.com

4 Graduação em Medicina da Faculdade de Ciências Médicas de Minas Gerais. ORCID: https://orcid.org/oooo-ooo3-2156-II84 E-mail: anabeatrizpedrosa@hotmail.com
} 


\section{INTRODUÇÃO}

O conhecimento da anatomia é de grande interesse na progressão da gangrena de Fournier ${ }^{1,2}$. A exposição dos testículos constitui um problema, devido à possibilidade de redução da espermatogênese, sobretudo em jovens. Diabetes mellitus e imunossupressão são os principais fatores de risco ${ }^{1,2,3}$. A fasciite necrosante resultante da gangrena de Fournier caracteriza-se pela isquemia e trombose dos vasos subcutâneos da região escrotal, resultando em necroses ${ }^{4,5,6}$, que necessitam de desbridamentos $7,8,9$ e exposição testicular. Os objetivos do estudo são descrever a técnica de tunelização do testículo exposto e a apresentar a importância da anatomia na gangrena de Fournier.

\section{Método}

\section{Técnica de Tunelização do Testículo Exposto:}

Para a tunelização do testículo exposto realizou-se ponto de reparo com fio de nylon monofilamentar 4.0 no testículo a ser tunelizado, com as extremidades dos fios longas. Puncionou-se com jelko no 18 a face medial e terço superior da coxa homolateral ao testículo exposto, introduzindo uma das extremidades longas do ponto de reparo do testículo no orifício do jelko (Figura I). Realizou-se movimento retrógrado com a remoção do jelko da coxa e a exteriorização de uma das extremidades longas do ponto de reparo do testículo sobre a pele da coxa. Nova punção foi realizada com o mesmo jelko $\mathrm{n}$ - $\mathrm{I} 6$ na face medial e terço superior da coxa homolateral ao testículo exposto, em topografia $5 \mathrm{~mm}$ inferior ao local da primeira punção, seguido da introdução da outra extremidade longa do ponto de reparo do testículo no orifício do jelko. Realizou-se a remoção do jelko da coxa e a exteriorização da outra extremidade do ponto de reparo do testículo sobre a pele da coxa. O testículo foi tracionado e tunelizado para a tela subcutânea da coxa homolateral, por meio da tração das extremidades dos fios exteriorizados na pele. Utilizando as extremidades dos fios do ponto de reparo do testículo realizou-se um ponto de sutura na pele da coxa. 


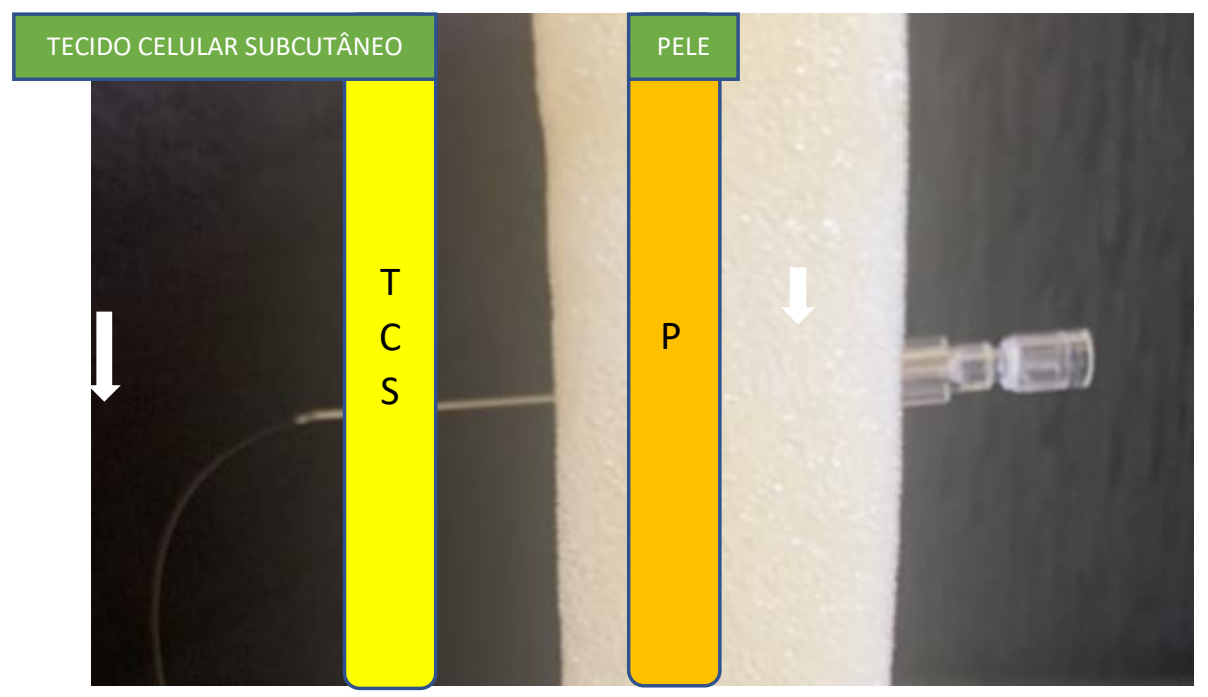

Figura I: A imagem é representativa da camada superficial, a pele (P), e da camada profunda, o tecido celular subcutâneo (TCS), da coxa. Observa-se que o jelko i6 (seta menor) transfixou a camada superficial (P) e a camada profunda (TCS) representadas na figura. Uma das extremidades do fio de mononylon 4.0, do ponto de reparo do testículo, foi introduzida no interior do jelko (seta maior).

\section{Importância da Anatomia na Gangrena de Fournier:}

Estudou-se a importância da anatomia na gangrena de Fournier por meio de avaliação criteriosa da literatura incluindo artigos científicos em base da dados Pubmed, BVS, Lilacs e livros consagrados na literatura. Os descritores utilizados foram: Gangrena de Fournier (Fournier Gangrene), Fasciite Necrosante (Fasciitis, Necrotizing), anatomia (anatomy), cirurgia geral (geral surgery) e cirurgia plástica (plastic surgery).

\section{Resultado}

\section{Técnica de tunelização do testículo exposto:}

A técnica de tunelização do testículo exposto (Figura 2) possibilitou o deslizamento e a tunelização do testículo para a tela subcutânea da coxa homolateral (Figura 3), por meio da tração das extremidades longas dos fios do ponto de reparo do testículo, que foram exteriorizados na pele, após a realização de movimento retrogrado do jelko 


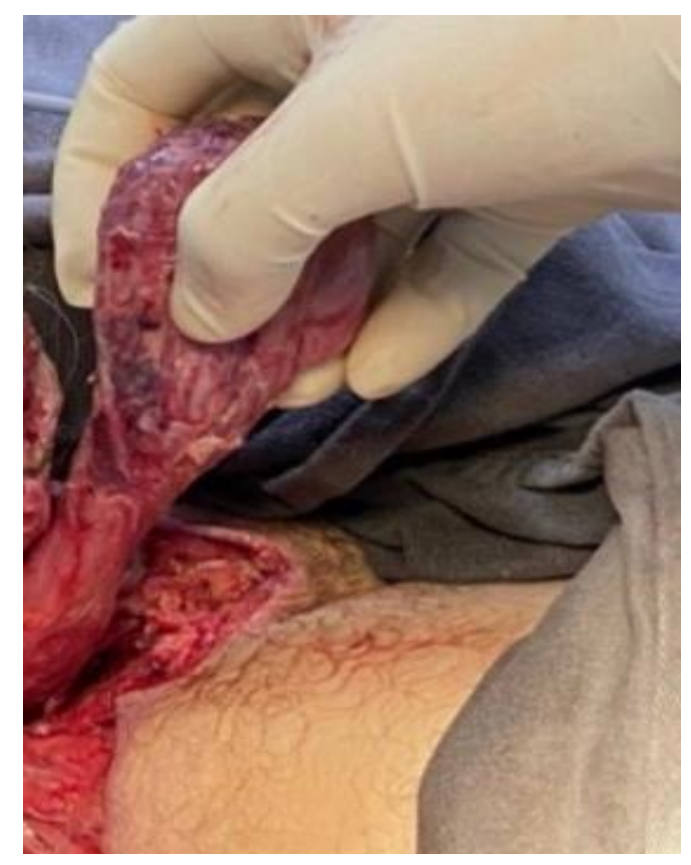

Figura 2: Testículo exposto (seta) após desbridamento de gangrena de Fournier.

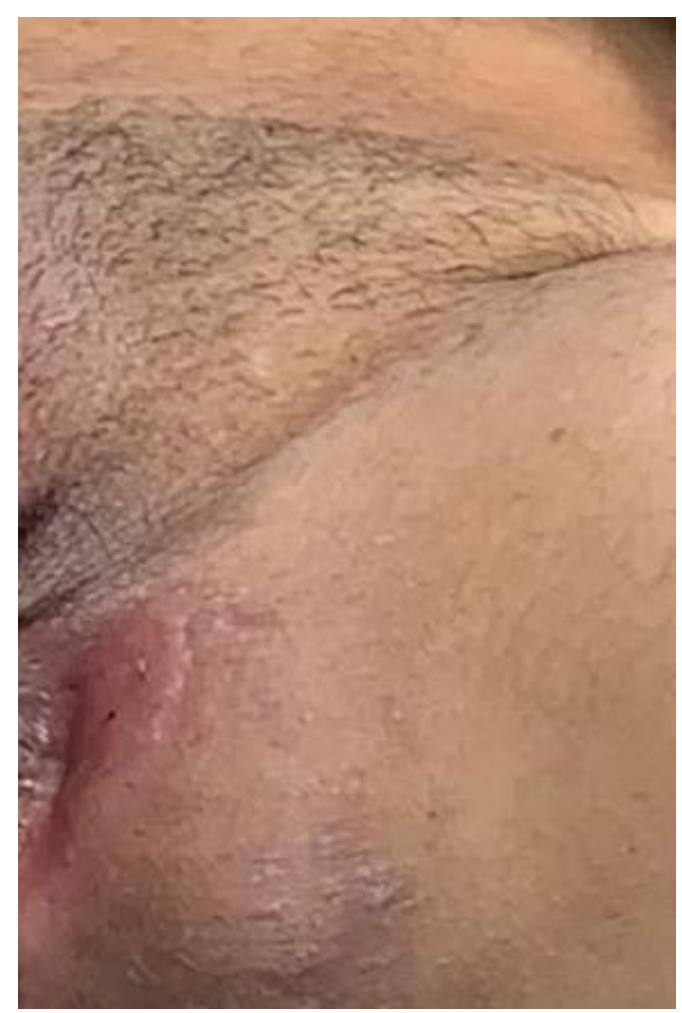

Figura 3: Testículo tunelizado na coxa (seta). 


\section{Importância da anatomia na gangrena de Fournier:}

O estudo da literatura realizado permitiu definir que as comunicações anatômicas existentes entre as camadas de revestimento das regiões perineal (Colles), peniana (Buck) e abdominal (Scarpa) e pênis (dartos) contribuem para a disseminação da infecção na gangrena de Fournier (Figura 4).

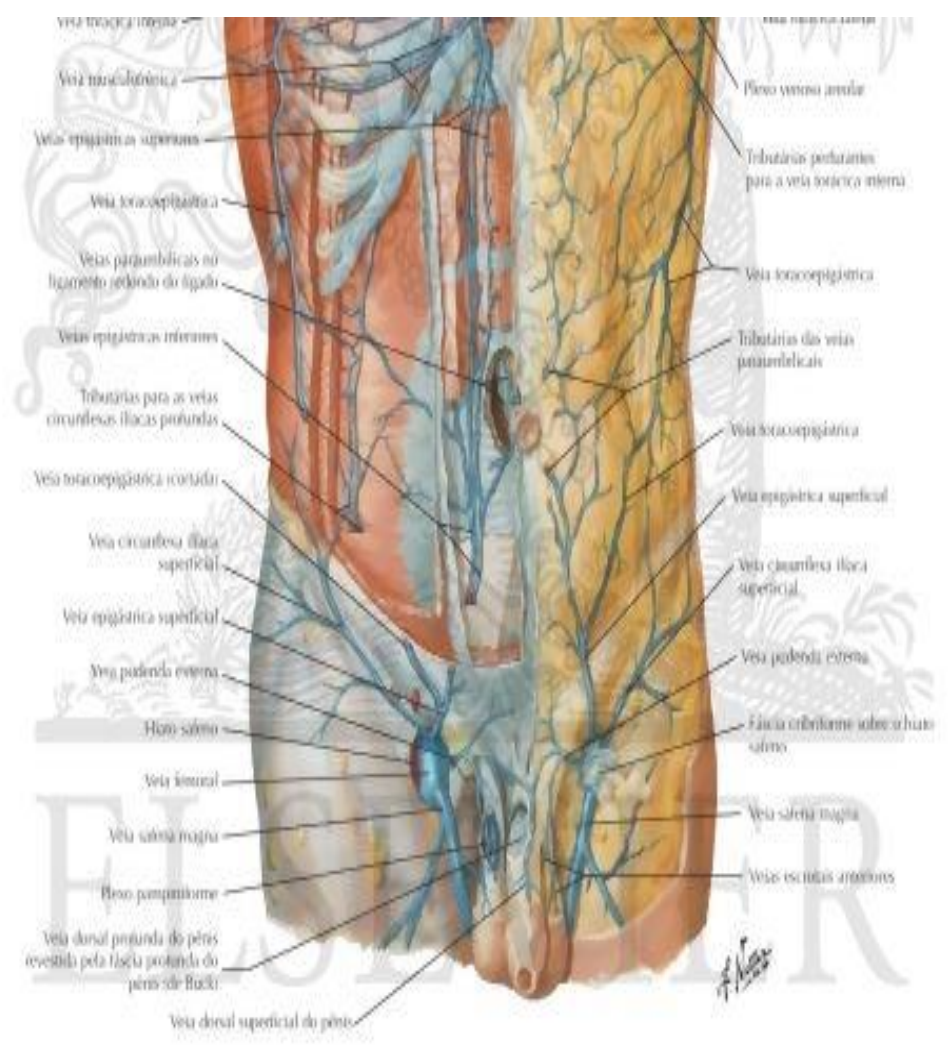

Figura 4: As comunicações anatômicas existentes entre as camadas de revestimentos do períneo, escroto, pênis e abdome favorecem a disseminação da infecção na gangrena de Fournier 5 .

\section{Discussão}

O conhecimento da anatomia é importante na gangrena de Fournier, pois a disseminação da infecção na fasciite necrosante ocorre, por meio da continuidade anatômica das camadas de revestimento. O escroto consiste em bolsa cutânea que contém os testículos e as partes inferiores do funículo espermático constituída por duas camadas, a pele, superficialmente, e o dartos, que, anatomicamente, consiste em uma camada de musculatura lisa, estando localizada sob a pele do escroto ${ }^{3,4}$. O dartos comunica-se com a fáscia muscular superficial do períneo denominada de fáscia de 
Colles, que reveste os músculos da porção superficial do períneo, enquanto a fáscia que reveste os corpos cavernosos do pênis recebe o nome de fáscia de Buck. A fáscia de Colles do períneo apresenta continuidade anatômica com a fáscia de Scarpa, camada de revestimento lamelar da parede do abdome ${ }^{4,5}$. A importante comunicação entre as camadas de revestimentos Colles, dartos, Buck e Scarpa é responsável pela progressão do processo infeccioso, iniciado na região períneo-escrotal, que dissemina para o pênis e a parede do abdome nos casos mais graves. A demora para definir o diagnóstico, o início tardio do tratamento ${ }^{7,8}$, diabetes mellitus e imunossupressão, $6,7,8$ são condições relacionadas ao aumento da mortalidade na gangrena de Fournier. Os métodos de imagem, tais como o ultrassom e a tomografia computadorizada, são importantes auxiliando no diagnóstico, ${ }^{9, \mathrm{ro}}$. A fisiopatologia da fasciite necrosante de Fournier caracteriza-se pela isquemia e trombose dos vasos, resultando em necrose da fáscia ${ }^{\mathrm{II}, \mathrm{I2}}$. Após a isquemia e trombose, ocorre a disseminação de bactérias, sendo as anaeróbicas produtoras de gases, responsáveis pela crepitação, encontrada nas primeiras 48 horas da infecção ${ }^{13}$, que pode se desenvolver sob a pele aparentemente normali4,15. Os microrganismos com maior prevalência são Escherichia coli, Staphylococcus aureus e Bacteróides fragilis e Streptococcus fecalis ${ }^{13,14,16,17}$. A redução da taxa de mortalidade depende do diagnóstico precoce e do início rápido dos tratamentos com antibióticos de amplo espectro ${ }^{18,19,20,21}$, desbridamentos dos tecidos necrosados, $22,23,24,25$ e a associação da terapia hiperbárica ${ }^{26,27,28,29}$. A exposição dos testículos poderá determinar a redução da espermatogênese. A posição do testículo tunelizado na face medial da coxa favorece a reconstrução com retalho, em outro tempo cirúrgico, pois quando o retalho fasciocutâneo da coxa for realizado para a reconstrução escrotal, ocorrerá o deslocamento do testículo tunelizado, juntamente com o retalho confeccionado ${ }^{30}$.

\section{Conclusão}

O estudo realizado permitiu concluir que a técnica de tunelização do testículo exposto possibilita o deslizamento do testículo para a tela subcutânea da coxa homolateral e que as comunicações anatômicas entre as camadas de revestimento do 
períneo, escroto, pênis e abdome contribuem para a disseminação da infecção na gangrena de Fournier.

\section{Referências}

I.Alcaraz FDA, Lozoya GAH, Uribe MJ, Romero MR, Gutierrez GA. Etiology of Fournier gangrene as a prognostic factor in mortality: analysis of I2I cases. Actas Urol Esp 2019;43(10):557-561.

2.Montrief T, Long B, Koyfman A, Auerbach J. Fournier Gangrene: a review for emergency clinicians. J Emerg Med 2019;57(4):488-500.

3.Fattini CA, Dângelo JG. Anatomia humana sistêmica e segmentar. $3^{\underline{a}}$ ed. Atheneu 2011. 780 p.

4.Moore KL, Dalley AF, Agur AMR. Anatomia orientada para clínica. 8 ed. Guanabara 2019. 1128p.

5. Netter FH. Atlas de anatomia humana 3D. 6ee. Elsevier 2015. 640p.

6. Short B. Fournier gangrene: an historical reappraisal. Intern Med J 2018;48(9):115760.

7. Singh A, Ahmed K, Aydin A, Khan MS, Dasgupta P. Fournier's gangrene. A clinical review. Arch Ital Urol Androl 2016;88(3):157-64.

8. Norton KS, Johnson LW, Perry T, Perry KH, Sehon JK, Zibari GB. Management of Fournier's gangrene: an eleven year retrospective analysis of early recognition, diagnosis, and treatment. Am Surg 2002;68(8):709-13.

9. Kuchinka J, Matykiewicz J, Wawrzycka I, Kot M, Karcz W, Głuszek S. Fournier's gangrene - challenge for surgeon. Pol Przegl Chir 2019;92(5):I-5.

Io. Eke N. Fournier's gangrene: a review of 1726 cases. Br J Surg 2000;87(6):718-28.

II. Arora A, Rege S, Surpam S, Gothwal K, Narwade A. Predicting Mortality in Fournier Gangrene and Validating the Fournier Gangrene Severity Index: Our Experience with 50 Patients in a Tertiary Care Center in India. Urol Int 2019;102(3):311I8.

12. Abass-Shereef J, Kovacs M, Simon EL. Fournier's Gangrene Masking as Perineal and Scrotal Cellulitis. Am J Emerg Med 2018;36(9):1719.

13. Ballard DH, Mazaheri P, Raptis CA, Lubner MG, Menias CO, Pickhardt PJ, Mellnick VM. Fournier Gangrene in Men and Women: Appearance on CT, Ultrasound, and MRI and What the Surgeon Wants to Know. Can Assoc Radiol J 2020;7I(I):30-9. 
I4. Levenson RB, Singh AK, Novelline RA. Fournier gangrene: role of imaging. Radiographics 2008;28(2):519-28.

15. Yilmazlar T, Gulcu B, Isik O, Ozturk E. Microbiological aspects of Fournier's gangrene. Int J Surg 2017;40(I):135-38.

16. Kuo CF, Wang WS, Lee CM, Liu CP, Tseng HK. Fournier's gangrene: ten-year experience in a medical center in northern Taiwan. J Microbiol Immunol Infect 2007;40(6):500-6.

17. Cardoso JB, Féres O. Revista da Faculdade de Medicina de Ribeirão Preto. 2007;40(4):493-9.

18. Kuzaka B, Wróblewska MM, Borkowski T, Kawecki D, Kuzaka P, Młynarczyk G, Radziszewski P. Fournier's Gangrene: Clinical Presentation of 13 Cases. Med Sci Monit 2018;28(24):548-55.

19. Demir CY, Yuzkat N, Ozsular Y, Kocak OF, Soyalp C, Demirkiran H. Fournier Gangrene: Association of Mortality with the Complete Blood Count Parameters. Plast Reconstr Surg 2018 Jul;142(I):68-75.

2o. Lin TY, Cheng IH, Ou CH, Tsai YS, Tong YC, Cheng HL, Yang WH, Lin YM, Cheng YS. Incorporating Simplified Fournier's Gangrene Severity Index with early surgical intervention can maximize survival in high-risk Fournier's gangrene patients. Int J Urol $2019 \mathrm{Jul} ; 26(7): 737-43$.

2I. Radcliffe RS, Khan MA. Mortality associated with Fournier's gangrene remains unchanged over 25 years. BJU Int 2020;125(4):610-16.

22. Fernandez-Alcaraz DA,Guillén-Lozoya AH, Uribe-Montoya J, Romero-Mata R, Gutierrez-González A. Etiology of Fournier gangrene as a prognostic factor in mortality: Analysis of I21 cases. Actas Urol Esp 2019;43(10):557-6r.

23. Carrillo-Córdova LD, Aguilar-Aizcorbe S, Hernández-Farías MA, AcevedoGarcía C, Soria-Fernández G, Garduño-Arteaga ML. Escherichia coli producer of extended-spectrum beta-lactamases as causative agent of Fournier gangrene of urogenital origin associated with higher mortality [Escherichia coli productora de betalactamasas de espectro extendido como agente causal de gangrena de Fournier de origen urogenital asociada a mayor mortalidad. Cir 2018;86(4):327-31.

24. Syllaios A, Davakis S, Karydakis L, Vailas M, Garmpis N, Mpaili E, Kyros E, Felekouras E, Papalampros A. Treatment of Fournier's Gangrene With Vacuumassisted Closure Therapy as Enhanced Recovery Treatment Modality. In Vivo 2020;34(3):I499-I502. 
25. Egin S, Kamali S, Hot S, Gökçek B, Yesiltas M. Comparison of Mortality in Fournier's Gangrene with the Two Scoring Systems. J Coll Physicians Surg Pak 2020;30(I):67-72.

26. Mindrup SR, Kealey GP, Fallon B. Hyperbaric oxygen for the treatment of fournier's gangrene. J Urol 2005;173(6):1975-7.

27. Li C, Zhou X, Liu LF, Qi F, Chen JB, Zu XB. Hyperbaric Oxygen Therapy as an Adjuvant Therapy for Comprehensive Treatment of Fournier's Gangrene. Urol Int 2015;94(4):453-8.

28. Rebeca CZ, Aquino C, Angela N, Zapata L, Massiel F; Tacusi M, et al. Impacto de la enfermedad de Fournier en pacientes del Hospital Clínico Viedma durante enero del 2008 a marzo del 2013 / The impact of Fournier's gangrene disease in patients at the Hospital Clínico Viedma during january 2008 - march 2013. Rev cientif cienc med 2013; 6(I): 17-19.

29 Ramírez C F, Ramírez OJ. Incidencia, factores de riesgo, mortalidad y protocolo de tratamiento quirúrgico de la gangrena de Fournier, hospital Luis Vernaza, período 2003 - 2008 / Incidence, risk factors, mortality, and surgical treatment protocol of Fournier's gangrene at the Luis Vernaza hospital period 2003 - 2008. Medicina (Guayaquil) 2011; 16(3): 183-8.

30. Mello DF, Júnior AH. Reconstrução escrotal com retalho fasciocutâneo superomedial da coxa. Rev. Col. Bras. Cir. 45(or)2or8:er389. 